\title{
The Methods of Process Planning Gas Treatment Facilities of a Power Plant
}

\author{
Olesya Aksenova ${ }^{1, *}$, Evgenia Nikolaeva ${ }^{1}$, and Riccardo Paulman ${ }^{2}$ \\ ${ }^{1}$ T.F. Gorbachev Kuzbass State Technical University, Underground Mining Department, 650000 \\ Kemerovo, Russian Federation \\ ${ }^{2}$ Queensland University of Technology, 2434 QLD 4001, Brisbane, Australia
}

\begin{abstract}
The article seeks to examine the efficiency of applying means of mathematical processing and computer modeling of the work processes of gas cleaning facilities of power plants to optimize the process of planning and designing of future power plant in operation. A way of solution of the gas outbursts cleaning problem at the stage of designing measures for the development of waste processing systems through mathematical processing of expected volumes of gas outbursts and computer simulation of individual pieces of equipment and working areas as a whole is offered. The authors present the results of the processing of the projected technological processes and the gas outbursts cleaning facilities in terms of E-networks and using mathematical processing in the application Simulink, which allows to build a model of the device and to carry out calculations on the screen using the library of building blocks. A $3 \mathrm{D}$ model of the gas cleaning facilities, which allows to visualize the technological process and to compare it with theoretical calculations at the design stage of the future power plant and, if necessary, make changes to the project, has been created by means of computer modeling.
\end{abstract}

\section{Introduction}

At present, the electric power industry is the most important basic industry of Russia. The level of its development affects the entire national economy, as well as the level of development of scientific and technological progress in the country.

It is practically impossible to imagine modern life without electric energy. The electric power industry has invaded all spheres of human activity: industry and agriculture, science and space, and our everyday life. In industrial applications, electric power is used both for activating various mechanisms and in technological processes directly.

Major electricity producers include hydroelectric power plants, nuclear power plants and thermal power plants. Thermal power plants (TPPs) require special attention. There are about 700 large and medium-sized thermal power plants in Russia. They produce up to $70 \%$ of electricity. TPPs use organic fuel - coal, oil, gas, fuel oil, shale oil, peat.

\footnotetext{
${ }^{*}$ Corresponding author: olesya aksenova42@mail.ru
} 
Thermal power plants are preferable compared to the others, as having a relatively free arrangement associated with wide distribution of fuel resources in Russia and are able to generate electricity without seasonal variation.

The future of mankind depends on the solution of two related problems, such as the need for energy development and the pollution of the TPP environment. Air, soil, water, plants and animals suffer from consumer attitudes to it. TPP pollution disrupts the natural functioning of all components of the biosphere.

TPP air pollution is a big problem. In terms of emissions of harmful substances into the atmosphere, thermal power plants occupy a leading place. That is almost thirty percent of the total emissions of all the enterprises in various industries. And this is more than six million tons of dust, harmful compounds of carbon, nitrogen, sulfur, vanadium and other elements of the periodic table.

If no action is taken, it leads to the destruction of the ozone layer of the Earth. Every year the percentage of people with allergies of varying severity is growing. The TPP air pollution is very dangerous for health and life of inhabitants of the cities. This is especially detrimental to the respiratory and cardiovascular systems of the body.

Not fulfilling the environmental requirements for thermal power plants at all the stages of construction and operation, people endanger their lives and the lives of future generations.

Therefore, when planning a future functioning power plant, special attention should be paid to the availability of gas treatment systems, namely gas emission treatment system and system of a complete cycle of ash and slag waste processing, which will not only ensure environmental and industrial safety, but will also be cost-effective for the enterprises and the country as a whole.

\section{Materials and Methods}

At the stage of planning the processes of fuel and energy facilities and the development of technical documentation, a modern range of computer programs enables to present an analysis of the future TPP operation in different ways. Mathematical modeling [8-10] makes it possible to verify the correctness of the model and its correction at the stage of construction, three-dimensional computer simulation allows to get a realistic image based on the available technical documentation, to identify technical errors and make adjustments at the planning stage of individual technological processes and thermal power plants as a whole. During the mathematical analysis it is advisable to use the E-network device $[1,2]$. As an extension of Petri nets, E-nets are a graphical and mathematical tool used to model systems of different types. The paper studies an abstracted model of thermal power plants with the possibility of expansion (Fig.1.).

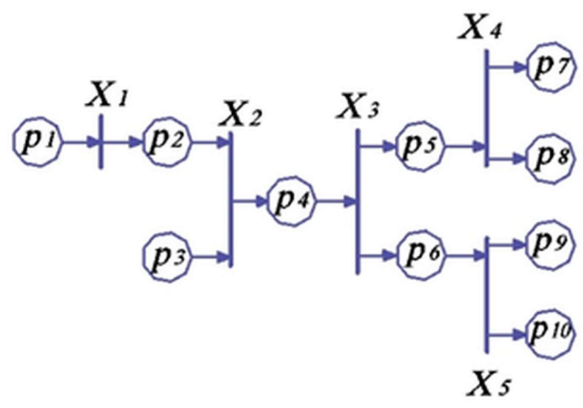

Fig. 1. The model CHP in terms of E-nets. 
where $p_{1}$ - a new portion of coal entered the crusher station;

$\mathrm{p}_{2}$ - coal is processed;

$\mathrm{p}_{3}$ - condensate and replenished water loss are at the storage facility;

$\mathrm{p}_{4}$ - the raw material is in the furnace chamber;

$\mathrm{p}_{5}$ - combustion products are obtained;

$\mathrm{p}_{6}$ - steam is supplied to the turbine;

$\mathrm{p}_{7}-$ bottom ash waste is transported to the recycling system;

$\mathrm{p}_{8}-$ gaseous wastes enter the cleaning system;

$\mathrm{p}_{9}$ - power is allocated to external consumers;

$\mathrm{p}_{10}$ - steam from the extraction turbine is supplied to CHP;

$\mathrm{X}_{1}=\left(\mathrm{T}, 0, \varphi_{1}\right)-$ the process of coal processing;

$$
\varphi_{1}=\mathrm{L}\left(\mathrm{X}_{1}\right)=\left\{\mathrm{p}_{1} \mathrm{X}_{1} \mid \mathrm{X}_{1} \rightarrow \mathrm{p}_{1} \mathrm{p}_{2}\right\}=\left\{\mathrm{p}_{1} \mathrm{p}_{2}\right\} ;
$$

$\mathrm{X}_{2}=\left(\mathrm{I}, 0, \varphi_{2}\right)$ - loading of raw materials into the furnace chamber;

$\varphi_{2}=L\left(X_{2}\right)=\left\{p_{2} p_{3} X_{2} \mid X_{2} \rightarrow p_{2} p_{3} p_{4}\right\}=>L\left(X_{2}\right)=\left\{p_{2} p_{3} p_{4}\right\}$

$\mathrm{X}_{3}=\left(\mathrm{F}, 0, \varphi_{3}\right)$ - processing of raw materials;

$\mathrm{X}_{4}=\left(\mathrm{F}, 0, \varphi_{4}\right)$-waste withdrawal;

$$
\varphi_{3}=\mathrm{L}\left(\mathrm{X}_{3}\right)=\left\{\mathrm{p}_{4} \mathrm{X}_{3} \mid \mathrm{X}_{3} \rightarrow \mathrm{p}_{4} \mathrm{p}_{5} \mathrm{p}_{6}\right\}=\left\{\mathrm{p}_{4} \mathrm{p}_{5} \mathrm{p}_{6}\right\}
$$

$$
\varphi_{4}=\mathrm{L}\left(\mathrm{X}_{4}\right)=\left\{\mathrm{p}_{5} \mathrm{X}_{4} \mid \mathrm{X}_{4} \rightarrow \mathrm{p}_{5} \mathrm{p}_{7} \mathrm{p}_{8}\right\}=\left\{\mathrm{p}_{5} \mathrm{p}_{7} \mathrm{p}_{8}\right\}
$$

$\mathrm{X}_{5}=\left(\mathrm{F}, 0, \varphi_{5}\right)-$ electricity generation and steam removal;

$\varphi_{5}=\mathrm{L}\left(\mathrm{X}_{5}\right)=\left\{\mathrm{p}_{6} \mathrm{X}_{5} \mid \mathrm{X}_{5} \rightarrow \mathrm{p}_{6} \mathrm{p}_{9} \mathrm{p}_{10}\right\}=\left\{\mathrm{p}_{6} \mathrm{p}_{9} \mathrm{p}_{10}\right\}$, the initial marking is represented by a vector: $(1,0,1,0,0,0,0,0,0,0)$.

To implement the computer-built model of the E-network [4] the technical computing application program package MatLab was used [1,3], namely, its application - Simulink [5].

\section{Results and Discussion}

When Simulink is used for simulation, the principle of visual programming is implemented, according to which the user creates a model of the device and performs calculations on the screen using the library of building blocks. To fulfil this task, unlike in case with the classic methods of modeling, the user does not need to thoroughly study the programming language and numerical methods of mathematics, but rather have general user skills and knowledge of the subject area in which he/she works.

Using subsystems and mechanism of their blocks allows to create the blocks that are not inferior to the standard ones in their design (own window of block parameters, icon, help, etc.).

The number of subsystems in the model is not limited, besides subsystems can include other subsystems. The level of nesting of subsystems in each other is not limited.

The model created in Simulink is a balance model of material flows [6,7], consisting of several technological stages: coal losses during its preparation for combustion; generation of electricity; formation and processing of bottom ash waste; formation and cleaning of gas outbursts. For the sake of clarity of the balance model and the convenience of its expansion, processing and cleaning units were implemented in the form of subsystems. Let us consider the subsystem of cleaning gas outbursts TPP, which in turn also consists of several modules: an electric filter, carbon capture, a system of purification from nitrogen oxides and a system of desulphurization.

As an example of mathematical processing and three-dimensional computer modeling, let's take a closer look at the nitrogen dioxide capture system.

Nitrogen dioxide is a component of the mixture of thermal power plant gas emissions, and it has a very detrimental effect on the environment and human health. It is second only 
to sulfur dioxide as for its contribution to the acidity of precipitation. Long-term exposure to nitrogen dioxide at concentrations of $470-1880 \mu \mathrm{g} / \mathrm{m} 3$ may inhibit the growth of some plants. In addition, nitrogen dioxide plays an important role in the formation of photochemical smog, which significantly worsens visibility and spoils the aesthetics of the environment as a whole.

When long inhaled, this gas produces oxygen starvation of tissues, especially in children. It causes diseases of blood circulation and malignant neoplasms. It is toxic, adversely affects genetic material, reproductive organs, eyes, skin. It has a strong effect on the central nervous system.

Among the variety of methods for reducing nitrogen oxide emissions, selective catalytic reduction is considered to be the most effective (Fig. 2). It has long been used in power plants in Europe, the USA and Japan.

Ammonia or urea is commonly used as a reducing agent and it is injected into the flue gas stream. Near the surface of the catalyst, reduction reactions of varying degrees of intensity take place, as a result of which nitrogen oxides turn into molecular nitrogen, which is a part of the air.

There are three main diagrams of the SCR-installation work. Let's consider the most expensive and effective of them - tail-end. It consists in the installation of the DENOX reactor after all the heating surfaces of the technological diagram (thence its name is derived "tail-end"). This option is more suitable for implementation on the boilers in action, as the catalyst is less exposed to mechanical wear, corrosion or deactivation.

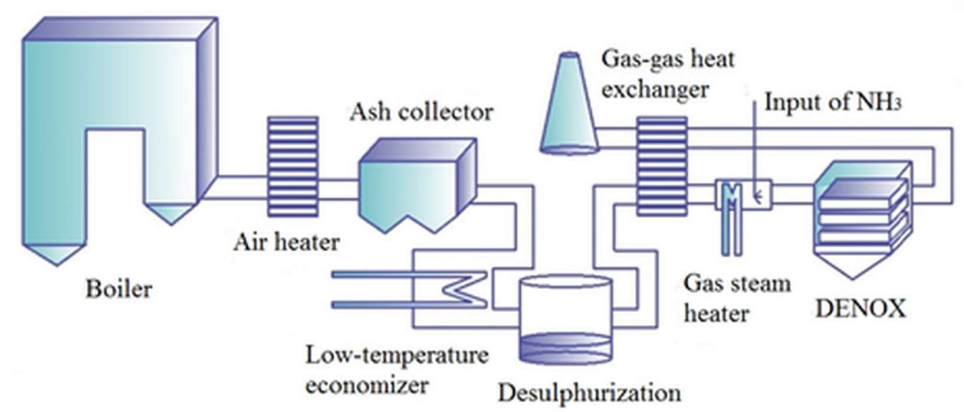

Fig. 2. The diagram of selective catalytic reduction process, including conversion of $\mathrm{NO}_{2}$ into molecular nitrogen.

The model of the cleaning from nitrogen oxides system, described with the help of Enetworks is presented in figure 3 .

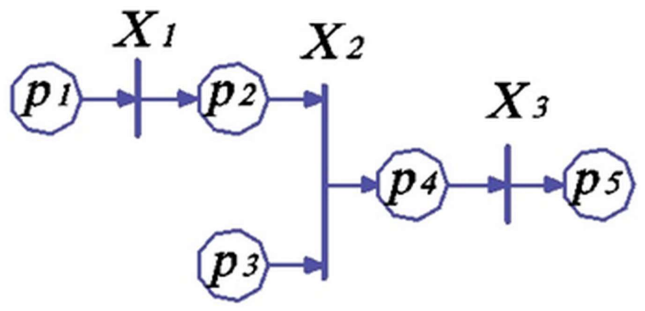

Fig. 3. The model of cleaning from nitrogen oxides system in terms of E-networks.

Introduce the notation:

$p_{1}$ - flue gases enter the heat exchanger;

$p_{2}$ - flue gases are put into the steam heater; 
$p_{3}-$ ammonia water from the storage gets to the steam heater;

$p_{4}$ - a mixture of the flue gases and ammonia enters the catalyst;

$p_{5}-$ the purified gas together with the formed molecular nitrogen is removed from the installation through the exhaust stack;

$X_{1}=\left(T, 0, \varphi_{1}\right)-$ gas heat treatment process;

$$
\varphi_{1}=L\left(X_{1}\right)=\left\{p_{1} X_{1} \mid X_{1} \rightarrow p_{1} p_{2}\right\}=\left\{p_{1} p_{2}\right\}
$$

$X_{2}=\left(I, 0, \varphi_{2}\right)$ - the process of injecting ammonia into the flue gas stream;

$$
\varphi_{2}=L\left(X_{2}\right)=\left\{p_{2} p_{3} X_{2} \mid X_{2} \rightarrow p_{2} p_{3} p_{4}\right\}=>L\left(X_{2}\right)=\left\{p_{2} p_{3} p_{4}\right\}
$$

$X_{3}=\left(T, 0, \varphi_{3}\right)-$ reduction reactions of different intensity.

$$
\varphi_{1}=L\left(X_{1}\right)=\left\{p_{4} X_{3} \mid X_{3} \rightarrow p_{4} p_{5}\right\}=\left\{p_{4} p_{5}\right\}
$$

The model created in Simulink is a balance model of material flows, in which the following technological stages were implemented:

1. The loss of coal during its preparation for combustion.

2. Generating of power.

3. Formation and processing of ash and slag waste.

4. Formation and purification of gas emissions.

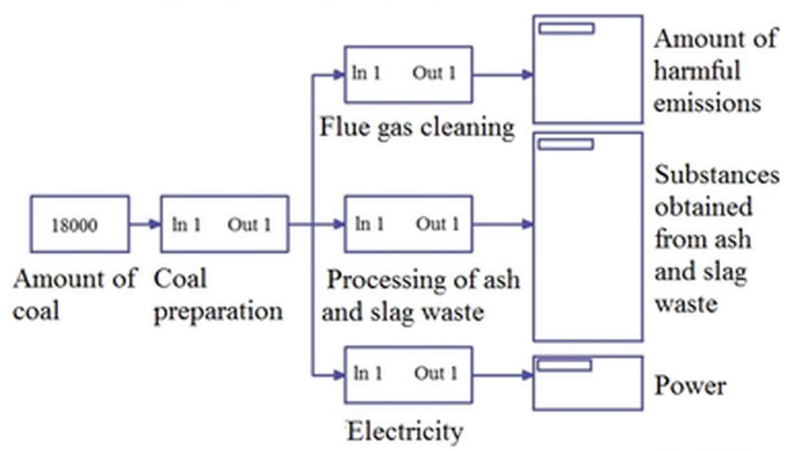

Fig. 4. Balance model of thermal power plants work in Simulink.

The model used the parameters of the average thermal power plant work with an efficiency close to $40 \%$, as well as the average amount of electricity generated by the combustion of 1 ton of conventional fuel.

For the purpose of clarity of the model and the simplicity of its extension, the units of processing and purification have been implemented in the form of subsystems.

Consider the module of the purification from nitrogen oxides system. Its implementation is shown in Fig. 5.

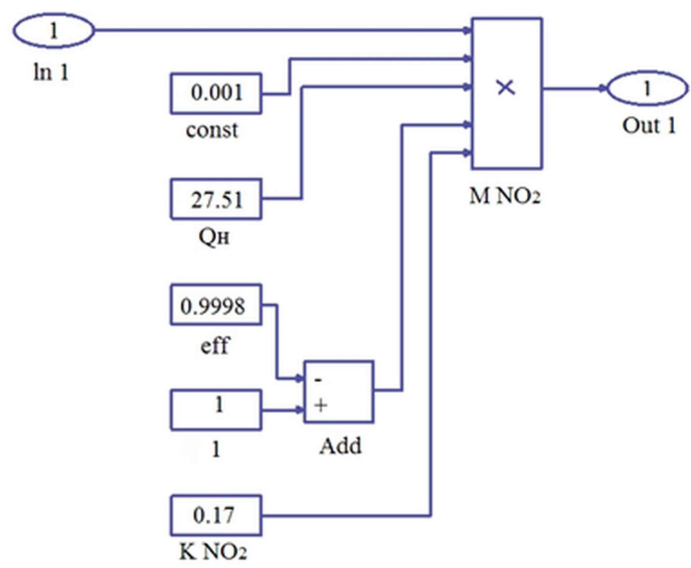


Fig. 5. The system of $\mathrm{NO}_{2}$ conversion into molecular nitrogen in Simulink.

To determine its characteristics the following formula was used:

$$
\mathrm{M}_{\mathrm{NO}_{2}}=\mathrm{m} \cdot \mathrm{Q}^{\mathrm{H}} \cdot \mathrm{K}_{\mathrm{NO}_{2}} \cdot(1-\beta) \cdot 10^{-3},
$$

where $\mathrm{M}_{\mathrm{NO}_{2}}$ - gross emission of nitrogen oxide expressed in terms of nitrogen dioxide; $\mathrm{Q}^{\mathrm{H}}$ - lower heat of natural fuel combustion, $\mathrm{MJ} / \mathrm{kg}$;

$\mathrm{K}_{\mathrm{NO}_{2}}$ - parameter characterizing the amount of nitrogen oxides formed per GJ of heat, $\mathrm{kg} / \mathrm{GJ}$;

$\beta-$ coefficient depending on the degree of reduction of nitrogen oxide emissions as a result of the application of technical solutions.

The amount of substances (in tons) formed in the processing of 18000 tons of coal: particulate matter - 0,5837; carbon dioxide - 105,4; nitrogen oxide $-0,01684$; sulfur oxides $-1,782$; rough gold and silver $(<10 \%)-0,00001818$; aluminium sulphate coagulant 2764; uranium $-6,48$; thorium 0,1163 ; tantalum, niobium, cerium, yttrium $-32,4$; titanium oxide $-170,5$; concentrate of zirconium with a hafnium - 1494; gallium - 0,02479; liquid glass - 3161; electricity (MW h) - 112540,06.

For realistic representation of the cleaning system of gas outbursts from nitrogen oxides and more exact calculation of the area planned under the equipment, on the basis of the available technical documentation solid models of the equipment and pipelines are constructed in several graphic editors. Then, taking into account the planned project area and the arrangement of the equipment the three-dimensional image of the future technological line and industrial process is realized (Fig.6) [1,2]. This image allows you to see the technical flaws in the theoretical planning and eliminate them.
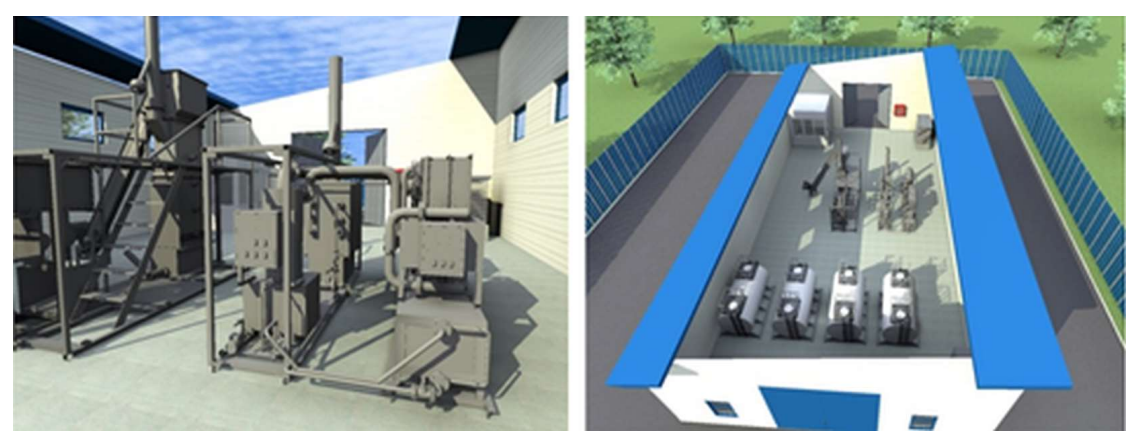

Fig. 6. Three-dimensional model of the system of $\mathrm{NO}_{2}$ conversion into molecular nitrogen.

\section{Conclusions}

The problem of the use of mathematical processing and computer simulation while planning the working processes of the gas cleaning facilities of a power plant at the stage of planning and design of TPP has been considered in the paper. Optimization of gas outbursts cleaning system planning by means of mathematical processing and computer simulation has been proposed, which can make it possible to evaluate the planned performance, select the production technology, as well as to ensure a high environmental effect of the planned waste treatment system. The paper presents the results of processing of the planned technological processes and gas outbursts cleaning system in terms of E-networks with the use of mathematical processing in the application Simulink, a three-dimensional model of the gas outbursts system has been built, which allows to visualize the process and compare with theoretical calculations at the design stage of a TPP and, if necessary, make changes. 


\section{References}

1. O. Aksenova, E. Nikolaeva, M. Cehlár, E3S Web Conf., 21, 03012 (2017)

2. O. Aksenova, E. Nikolaeva, E3S Web of Conf., 41, 01017 (2018)

3. M.I. Shilyaev, A.R. Bogomolov, I.V. Dvorovenko, A.S. Sysolyatin S.V. Kryukov, M.A. Chemakin, Coal in the 21st Century: Mining, Processing and Safety (Atlantis Press, Qingdao, 2016)

4. G.A. Kazunina, D.V. Alekseev, Advanced Materials Research, 933, 62 (2014)

5. A. Kovalchuk, M. Klopot, EEC, 3, 97-100 (2014)

6. M. Behandish, T. Horea, Computer-Aided Design, 70, 100 (2016)

7. Ba Wenlan, Ren Ning, Cao Lixin, Graphical Models, 82, 1 (2015) 